\title{
Comparison of radiographic sagittal spinopelvic alignment between skeletally immature and skeletally mature individuals with Scheuermann's disease
}

\author{
Marcin Tyrakowski $\cdot$ Piotr Janusz $\cdot$ Steven Mardjetko • \\ Tomasz Kotwicki $\cdot$ Kris Siemionow
}

Received: 5 May 2014/Revised: 23 September 2014/ Accepted: 23 September 2014/Published online: 4 October 2014

(C) The Author(s) 2014. This article is published with open access at Springerlink.com

\begin{abstract}
Purpose The aim of this study was to compare radiographic sagittal spinopelvic parameters between skeletally immature and skeletally mature patients with Scheuermann's disease (SD).

Methods Cross-sectional analysis of standing posteroanterior and lateral radiographs of the spine of patients with SD was performed. Sagittal vertical axis (SVA), thoracic kyphosis (TK), thoracolumbar kyphosis (TLK), lumbar lordosis (LL), pelvic incidence (PI), pelvic tilt (PT), and sacral slope (SS) were measured on the lateral radiographs. Risser's sign was assessed on the postero-anterior radiographs. All of the parameters measured were compared between skeletally immature (Risser's sign 0-3) versus mature patients (Risser's sign 5). PI, PT, and SS in both groups were compared to PI, PT, and SS reported for normal children, adolescents, and adults.
\end{abstract}

\footnotetext{
M. Tyrakowski ( $\square)$

Department of Orthopedics, Pediatric Orthopedics and Traumatology, The Centre of Postgraduate Medical Education in Warsaw, Konarskiego 13, 05-400 Otwock, Poland

e-mail: marcintyrak@gmail.com
}

M. Tyrakowski · K. Siemionow

Department of Orthopedic Surgery, University of Illinois at Chicago, 835 South Wolcott Ave, Room E-270, Chicago,

IL 60612, USA

P. Janusz $\cdot$ T. Kotwicki

Spine Disorders Unit, Department of Pediatric Orthopedics and Traumatology, University of Medical Sciences in Poznan, Ul. 28 Czerwca 1956 roku nr 135, 61-545 Poznan, Poland

S. Mardjetko

Illinois Bone and Joint Institute, 9000 Waukegan Road,

Morton Grove, IL 60053, USA
Results Sixty-six patients with SD (33 immature and 33 mature) were retrospectively reviewed and included in the study. There was no significant difference between the two groups of SD patients in: SVA (-16.6 vs. $-22.9 \mathrm{~mm}$, $p=0.74)$, TK $\left(57.8^{\circ}\right.$ vs. $\left.56^{\circ}, p=0.66\right)$, TLK $\left(7.8^{\circ}\right.$ vs. $\left.11.7^{\circ}, p=0.14\right)$, LL (63. $2^{\circ}$ vs. $\left.62.2^{\circ}, p=0.74\right)$, PI $\left(36.7^{\circ}\right.$ vs. $\left.39.4^{\circ}, p=0.20\right)$, $\mathrm{PT}\left(3.8^{\circ}\right.$ vs. $\left.7.3^{\circ}, p=0.10\right)$, and SS $\left(32.8^{\circ}\right.$ vs. $\left.32.1^{\circ}, p=0.75\right)$. Both, the immature and mature group of SD patients presented significantly lower PI and SS than normal children, adolescents, and adults, and significantly lower PT than normal adults.

Conclusions There is no significant difference in sagittal spinopelvic parameters between skeletally immature and mature subjects with SD. Pelvic incidence in both groups of SD patients was significantly lower than PI in normal children, adolescents, and adults. This challenges the role of PI in predicting desired LL in patients with SD.

Keywords Sagittal spinopelvic alignment . Scheuermann's disease · Pelvic incidence . Thoracic kyphosis · Risser's sign

\section{Introduction}

A rigid kyphosis of the thoracic or thoracolumbar spine occurring in adolescence was first described by Scheuermann in 1920 [1]. Today Scheuermann's disease (SD) or Scheuermann's kyphosis is the most likely cause of structural hyperkyphosis in the pediatric population [2]. Two patterns of SD have been described, namely the more commonly occurring typical (thoracic) pattern, characterized by nonstructural hyperlordosis of the cervical and lumbar spine and the atypical (thoracolumbar) pattern that is thought to be more likely to progress in adulthood [2-4]. 
The etiology of SD is thought to be multifactorial and remains unknown [2, 4].

The double S shape of the human spine in sagittal plane is one of the evolutionary adaptations to bipedal locomotion, providing a compromise between stability and mobility [5]. Several quantitative parameters, both positional and anatomic, concerning the sagittal balance have been distinguished [6]. Pelvic incidence (PI) first introduced by Duval-Beaupère et al. is the most widely used anatomic parameter in assessing spinopelvic alignment [7]. Pelvic incidence is hypothesized to be the fundamental pelvic parameter for three-dimensional regulation of spinal sagittal curves [8]. It is accepted that PI becomes fixed with skeletal maturation and remains constant in adulthood, unless there is a pathological process that can modify the shape of the pelvis $[6,9,10]$. Deviations in pelvic morphology and subsequently higher values of PI were found to be associated with spondylolisthesis and idiopathic scoliosis [11-16]. Significantly lower PI was reported in patients with post-tuberculosis or congenital thoracic and thoracolumbar kyphosis [17]. Jiang et al. [18] reported significantly lower PI in SD adolescents than in healthy age-matched group. We have previously described significantly lower PI in skeletally mature patients with SD than what has been reported for healthy adults [19]. Both of the above-mentioned studies revealed that PI was lower in individuals with the thoracolumbar form of SD than in those with the thoracic form $[18,19]$. There are currently no published studies comparing PI and other parameters characterizing sagittal spinopelvic alignment in skeletally immature versus skeletally mature patients with SD. Such an analysis could be meaningful regarding potential etiology or compensatory mechanisms in obtaining and maintaining neutral sagittal balance in individuals with SD.

The aim of this study was to compare the radiographic sagittal spinopelvic parameters between skeletally immature and skeletally mature patients with SD.

\section{Materials and methods}

\section{Subjects}

After having obtained institutional review board's approval, a group of patients with SD examined between January 2005 and November 2013 with standard standing long-cassette postero-anterior and lateral digital radiographs taken at index presentation was retrospectively reviewed and included in the study. The diagnosis of SD was based on the radiological criteria reported by Sorensen (at least three consecutive vertebral bodies with a minimum of $5^{\circ}$ of wedging) with additional findings (irregularities of the vertebral endplates, disc material herniation through the endplates (Schmorl's nodes), narrowing of the disc spaces, and lengthening of the vertebral bodies) [20,21]. Similar radiologic protocol was used during the entire study period. Lateral radiographs were obtained with each subject in a comfortable standing position and knees fully extended with arms in slight forward flexion and forearms extended and resting on a support [22]. Patients with Risser's sign of $0,1,2$, or 3 were classified as skeletally immature ("immature group") and those with Risser's sign of 5 were classified as skeletally mature ("mature group") [23]. All of the patients with Risser's sign of 4 , those with other spinal pathologies besides SD (e.g., spondylolysis, spondylolisthesis, scoliosis, spina bifida occulta) and individuals whose radiograph quality was not sufficient to assess all the quantitative parameters were excluded from this study.

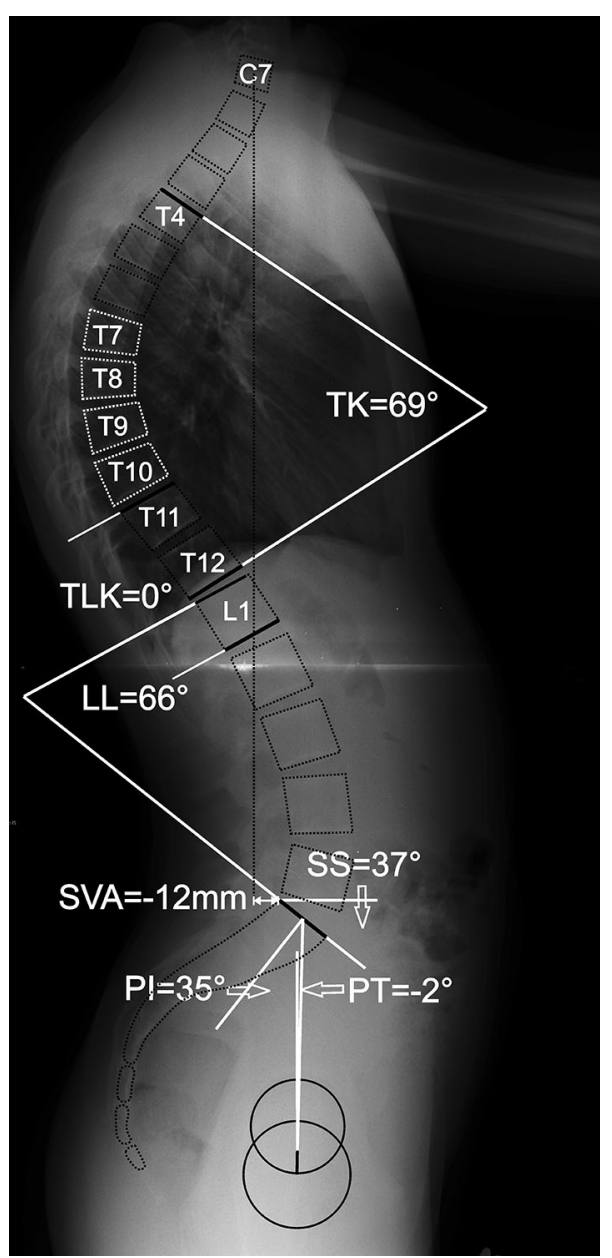

Fig. 1 Standing lateral radiograph of skeletally immature patient (Risser's sign of 2) with the typical form of Scheuermann's disease (apex of kyphosis located at the level of T8 vertebra). Anterior wedging of four consecutive vertebral bodies (T7-T10). TK thoracic kyphosis, TLK thoracolumbar kyphosis, $L L$ lumbar lordosis, $P I$ pelvic incidence, $P T$ cpelvic tilt, $S S$ sacral slope, $S V A$ sagittal vertical axis 
Radiographic analysis

All of the radiographs were reviewed by an experienced orthopedic spine surgeon using Surgimap Spine Software (Surgimap, New York, USA).

On each lateral radiograph 4 spinal parameters were measured, Fig. 1. Sagittal vertical axis (SVA) was defined as the linear horizontal distance between the C7 plumb line (originating in the center of $\mathrm{C} 7$ vertebral body) and the posterior corner of S1 endplate. Negative SVA was noted when the C7 plumb line was posterior to the $\mathrm{S} 1$ posterior corner. When the $\mathrm{C} 7$ plumb line was anterior to the S1 posterior corner the SVA was defined as positive. T4-T12 thoracic kyphosis (TK) was the angle measured between the proximal endplate of $\mathrm{T} 4$ vertebra and the distal endplate of the T12 vertebra using the Cobb's method. T11-L1 thoracolumbar kyphosis (TLK) was the angle measured between the proximal endplate of the T11 vertebra and the distal endplate of the L1 vertebra using the Cobb's method. Negative values of TLK indicated lordosis. L1-S1 lumbar lordosis (LL) was defined as the angle between the proximal endplate of the L1 vertebra and the endplate of S1 measured with Cobb's method.

On each lateral radiograph 3 pelvic parameters were measured, Fig. 1. Pelvic incidence (PI) was defined as the angle between the line joining the center of the bicoxofemoral axis and the center of the S1 endplate and the line perpendicular to the S1 endplate. Pelvic tilt (PT) was the angle between the line joining the center of the bicoxofemoral axis and the center of the S1 endplate and the vertical line. Negative values of PT were noted when the midpoint of S1 endplate was anterior to the center of bicoxofemoral axis. Sacral slope (SS) was defined as the angle between the line parallel to the S1 endplate and the reference horizontal line.

On each postero-anterior radiograph, the Risser's sign was assessed according to the original Risser's method [23].

Age, sex, and form of SD as well as the values of all of the parameters measured were compared between the mature and immature group.

The values of PI, PT, and SS of both, the immature and the mature patients with SD were compared to PI, PT, and SS reported for the largest groups of normal children, adolescents, and adults [24, 25].

All of the measurements were repeated by the same researcher after 1 month on 46 randomly chosen radiographs. The intraobserver reproducibility of all of the measurements performed was tested and quantified by the intraclass correlation coefficient (ICC) and a median error for a single measurement (SEM) [26].
Statistical analysis

The data was analyzed using the JMP 10.0.2 (SAS Institute Inc, Cary, NC) statistical software and in Microsoft Office Excel 2007 (Microsoft, Redmond, WA). Normal distribution of data was analyzed by use of the Shapiro-Wilk test. The unpaired $t$ test or the Wilcoxon's test was used to test the differences for the continuous data. The Fisher's exact test was used to test differences for the categorical data. The $p$ level of 0.05 was considered significant. A power of the $t$ test and of the Wilcoxon's test was set at 0.95 and the least significant value (LSV) was calculated for each comparison.

To estimate the sample size needed to test the intraobserver reproducibility of all of the measurements we treated

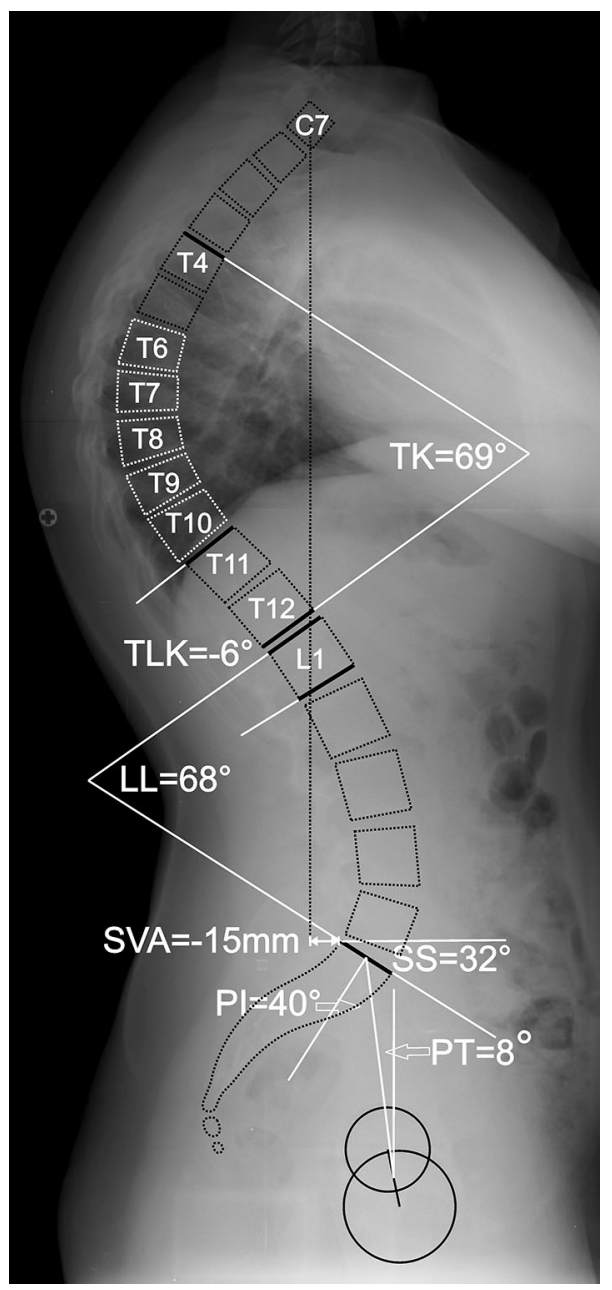

Fig. 2 Standing lateral radiograph of skeletally mature patient (Risser's sign of 5) with the typical form of Scheuermann's disease (apex of kyphosis located at the level of $\mathrm{T} 7$ vertebra). Anterior wedging of five consecutive vertebral bodies (T6-T10). TK thoracic kyphosis, $T L K$ thoracolumbar kyphosis, $L L$ lumbar lordosis, $P I$ pelvic incidence, $P T$ pelvic tilt, $S S$ sacral slope, $S V A$ sagittal vertical axis 
Table 1 Comparison of radiographic sagittal spinopelvic parameters between immature and mature patients with Scheuermann's disease

\begin{tabular}{|c|c|c|c|c|}
\hline Parameter & $\begin{array}{l}\text { Immature patients }(N=33) \\
\text { mean } \pm \mathrm{SD} \text { (range) }\end{array}$ & $\begin{array}{l}\text { Mature patients }(N=33) \\
\text { mean } \pm \mathrm{SD}(\text { range })\end{array}$ & $p$ & LSV \\
\hline SVA (mm) & $-16.6 \pm 23.8(-56-58)$ & $-22.9 \pm 40.7(-128-51)$ & $0.74^{t}$ & 18.3 \\
\hline $\mathrm{TK}\left({ }^{\circ}\right)$ & $57.8 \pm 15.3(3-81)$ & $56 \pm 18.8(13-96)$ & $0.66^{\mathrm{t}}$ & 8.4 \\
\hline $\operatorname{TLK}\left({ }^{\circ}\right)$ & $7.8 \pm 10.9(-8-45)$ & $11.7 \pm 12.6(-6-41)$ & $0.14^{\mathrm{w}}$ & 5.8 \\
\hline $\operatorname{LL}\left({ }^{\circ}\right)$ & $63.2 \pm 11.1(35-84)$ & $62.2 \pm 14.6(30-96)$ & $0.74^{t}$ & 6.4 \\
\hline PI $\left(^{\circ}\right)$ & $36.7 \pm 8.1(15-51)$ & $39.4 \pm 8.9(19-65)$ & $0.20^{\mathrm{t}}$ & 4.2 \\
\hline PT $\left({ }^{\circ}\right)$ & $3.8 \pm 7.5(-10-17)$ & $7.3 \pm 9.4(-10-33)$ & $0.10^{t}$ & 4.2 \\
\hline $\mathrm{SS}\left({ }^{\circ}\right)$ & $32.8 \pm 9.2(9-49)$ & $32.1 \pm 9.2(9-49)$ & $0.75^{\mathrm{t}}$ & 4.5 \\
\hline
\end{tabular}

$S D$ standard deviation, $S V A$ sagittal vertical axis, $T K$ thoracic kyphosis, $T L K$ thoracolumbar kyphosis, $L L$ lumbar lordosis, $P I$ pelvic incidence, $P T$ pelvic tilt, $S S$ sacral slope, $p$ indicates significance of the difference assessed by unpaired $t$ test (t) or Wilcoxon's test (w), $L S V$ least significant value detected by the $t$ test or the Wilcoxon's test with a power of 0.95

the ICC value greater than 0.7 (with its $95 \%$ confidence interval of $0.55-0.85$ ) as acceptable reproducibility for a research tool [27]. Thus, the minimum number of subjects to test the intraobserver reproducibility ( 2 series of measurements performed by 1 researcher) was 46 [28]. The ICC value of less than 0.40 indicated poor reproducibility, $0.40-0.75$ indicated fair to good agreement/reproducibility/ reliability, and values greater than 0.75 reflected excellent reproducibility [29].

\section{Results}

\section{Subjects}

Thirty-three consecutive skeletally immature patients with SD without prior treatment met the inclusion criteria. There were 26 patients with the typical form of SD (apex of the kyphosis at T6-T9 vertebra) and 7 patients with the atypical form of SD (apex of the kyphosis at T10-L2 vertebra), Fig. 1. Skeletally mature patients with SD were selected to match those immature in form of SD. Thirty-three mature patients with SD met the inclusion criteria (25 with the typical and 8 with the atypical form of SD), Fig. 2. Thus, the total number of subjects recruited for this study was 66 .

There were 17 patients with Risser's sign of 0 ( 5 females and 12 males), 4 patients with Risser's sign of 1 (all males), 5 patients with Risser's sign of 2 ( 2 females and 3 males), and 7 patients with Risser's sign of 3 (1 female and 6 males).

The mean age in the immature group was $14.1 \pm 1.4$ years (range 11-16.3 years) versus $22.7 \pm 7.6$ years (range 16.1-47.4 years) in the mature group with significant difference between the groups $(p<0.0001)$.

There were 8 females and 25 males in the immature group, and 11 females and 22 males in the mature group. The difference in sexes between the groups was insignificant $(p=0.29)$.
No statistically significant difference in form of SD between the two groups of patients were noted $(79 \%$ subjects (26/33) with the typical form in the immature group versus $76 \%(25 / 33)$ with the typical form in the mature group $(p=0.72)$ ).

\section{Radiographic analysis}

The mean, standard deviation, and range for the sagittal spinopelvic parameters for both the mature and the immature patient groups, together with $p$ values and LSVs from statistical tests comparing them are presented in Table 1. None of the parameters differ significantly between the two groups of patients with SD.

Comparisons of PI, PT, and SS in the immature and mature SD patients versus PI, PT, and SS reported for healthy children, adolescents, and adults are presented in Tables 2 and 3, respectively. Both groups of SD patients presented with significantly lower PI and SS compared to normal children, adolescents, and adults and significantly lower PT than normal adults. The difference in PT was insignificant when comparing both SD groups and normal children as well as skeletally mature patients with SD and healthy adolescents.

The ICC for intraobserver reproducibility of the measurements of each parameter was $\geq 0.9$ with the following SEMs for the particular parameters: SVA: $6.1 \mathrm{~mm}$; TK: 1.9 $9^{\circ}$ TLK: $1.0^{\circ}$; LL: $3.3^{\circ}$; PI: $1.2^{\circ}$; PT: $1.9^{\circ}$ and SS: $2.5^{\circ}$.

\section{Discussion}

We present a comparison of the sagittal spinopelvic alignment between the skeletally immature and mature patients with SD. We also compare PI, PT, and SS of these 2 groups of SD individuals with PI, PT, and SS reported for healthy children, adolescents, and adults. Such analyses have never been published. Jiang et al. [18] analyzed 
Table 2 Comparison of pelvic incidence, pelvic tilt, and sacral slope between skeletally immature patients with Scheuermann's disease versus historical normal children, adolescents, and adults

\begin{tabular}{|c|c|c|c|c|c|c|c|}
\hline \multirow[t]{2}{*}{ Study } & \multirow[t]{2}{*}{ Sample size } & \multicolumn{2}{|l|}{$\mathrm{PI}\left({ }^{\circ}\right)$} & \multicolumn{2}{|l|}{$\mathrm{PT}\left({ }^{\circ}\right)$} & \multicolumn{2}{|l|}{$\operatorname{SS}\left({ }^{\circ}\right)$} \\
\hline & & Mean \pm SD & $p$ & Mean \pm SD & $p$ & Mean \pm SD & $p$ \\
\hline Current study & 33 & $36.7 \pm 8.1$ & & $3.8 \pm 7.5$ & & $32.8 \pm 9.2$ & \\
\hline Normal children [24] & 167 & $43.7 \pm 9.0$ & $<0.0001 *$ & $5.5 \pm 7.6$ & 0.118 & $38.2 \pm 7.7$ & $0.001 *$ \\
\hline Normal adolescents [24] & 479 & $46.9 \pm 11.4$ & $<0.0001 *$ & $7.7 \pm 8.3$ & $0.002^{*}$ & $39.1 \pm 7.6$ & $<0.0001 *$ \\
\hline Normal adults [25] & 300 & $54.7 \pm 10.6$ & $<0.0001^{*}$ & $13.2 \pm 6.1$ & $<0.0001^{*}$ & $41.2 \pm 8.4$ & $<0.0001 *$ \\
\hline
\end{tabular}

$P I$ pelvic incidence, $P T$ pelvic tilt, $S S$ sacral slope, $S D$ standard deviation, $p$ indicates significance of the difference in PI, PT, or SS between the current study and the data from literature assessed by unpaired $t$ Student test

* Indicates statistically significant difference

Table 3 Comparison of pelvic incidence, pelvic tilt, and sacral slope between skeletally mature patients with Scheuermann's disease versus historical normal children, adolescents, and adults

\begin{tabular}{|c|c|c|c|c|c|c|c|}
\hline \multirow[t]{2}{*}{ Study } & \multirow[t]{2}{*}{ Sample size } & \multicolumn{2}{|l|}{$\mathrm{PI}\left({ }^{\circ}\right)$} & \multicolumn{2}{|l|}{$\mathrm{PT}\left({ }^{\circ}\right)$} & \multicolumn{2}{|l|}{$\operatorname{SS}\left({ }^{\circ}\right)$} \\
\hline & & Mean \pm SD & $p$ & Mean \pm SD & $p$ & Mean \pm SD & $p$ \\
\hline Current study & 33 & $39.4 \pm 8.9$ & & $7.3 \pm 9.4$ & & $32.1 \pm 9.2$ & \\
\hline Normal children [24] & 167 & $43.7 \pm 9.0$ & $0.006^{*}$ & $5.5 \pm 7.6$ & 0.151 & $38.2 \pm 7.7$ & $<0.0001 *$ \\
\hline Normal adolescents [24] & 479 & $46.9 \pm 11.4$ & $<0.0001 *$ & $7.7 \pm 8.3$ & 0.406 & $39.1 \pm 7.6$ & $<0.0001 *$ \\
\hline Normal adults [25] & 300 & $54.7 \pm 10.6$ & $<0.0001 *$ & $13.2 \pm 6.1$ & $<0.0001 *$ & $41.2 \pm 8.4$ & $<0.0001^{*}$ \\
\hline
\end{tabular}

$P I$ pelvic incidence, $P T$ pelvic tilt, $S S$ sacral slope; $p$ indicates significance of the difference in PI, PT, or SS between the current study and the data from literature assessed by unpaired $t$ Student test

* Indicates statistically significant difference

sagittal spinopelvic alignment in adolescents with SD and made a comparison with age-matched normal group. However, the authors considered chronological age of the subjects with no information regarding their skeletal maturity. We have previously presented sagittal spinopelvic alignment of skeletally mature patients with SD and compared them to historically published normal individuals [19]. In the current study, we present a comparison of the sagittal spinopelvic alignment between 2 groups of SD individuals, namely those that are skeletally immature (Risser's sign of $0,1,2$, or 3 ) to those who are skeletally mature (Risser's sign of 5). Since PI has been reported to increase during childhood and adolescence and to remain constant when skeletal maturity is reached $[6,9,10]$, we find it important to consider the skeletal maturity in such analyses. All of the patients with Risser's sign of 4 were excluded from the current study, because of data inconsistency: although Risser's sign of 4 has been thought as an indicator of vertebral growth cessation, there are reports emphasizing that at this stage of maturation some residual growth activity remains, and thus a potential for changes in pelvic morphology exists [30-32].

No significant differences in any of the sagittal spinal parameters analyzed (SVA, TK, TLK, and LL) were found between immature and mature patients with SD. Similarly, the 2 groups did not differ significantly between each other in regard to the type of SD (typical or atypical) and sex, making comparison of pelvic parameters between these two groups valuable.

The differences in PI, PT, and SS between the immature and mature individuals with SD were insignificant. Moreover, we found that PI in both SD groups $\left(36.7^{\circ}\right.$ and $39.4^{\circ}$ for the immature and mature, respectively) was significantly lower than those reported for healthy children $\left(43.7^{\circ}\right)$, adolescents $\left(46.9^{\circ}\right)$, and adults $\left(54.7^{\circ}\right)$ [24, 25]. Similarly, SS in both SD groups $\left(32.8^{\circ}\right.$ and $32.1^{\circ}$ for the immature and mature, respectively) was significantly lower than SS reported for healthy children $\left(38.2^{\circ}\right)$, adolescents $\left(39.1^{\circ}\right)$, and adults $\left(41.2^{\circ}\right)$ [24, 25]. Pelvic incidence has been proposed to be the fundamental pelvic parameter for three-dimensional regulation of the spinal sagittal curves [8]. Legaye et al. [8] stated that spinal balance is obtained as the result of optimal lordotic positioning of the vertebrae above a correctly oriented pelvis. Our results as well as the findings of Li et al. [17], Jiang et al. [18], and Tyrakowski et al. [19] suggest that first the pelvic orientation and next the morphology (PI) might be influenced by the sagittal spinal curves, thus serving as a compensatory mechanism for obtaining and maintaining a neutral sagittal balance. We hypothesize that an increased TK or TLK in patients with SD is compensated by increased LL to reach a neutral sagittal balance. When the natural reserve of increasing LL 
is maximized, any further compensation for the increasing TK or TLK may be accomplished by posterior tilting of the pelvis that decreases SS and with fixed PI increases PT. Increased PT that results in a posterior shift of the center of gravity in relation to the hip joints is biomechanically disadvantageous [33-35]. We suspect that in a growing individual, it can result in modification of the pelvis shape, so the PT increase is prevented. Thus, decreasing the PI could compensate for a decrease in SS with no changes in PT. Such a cause-effect relationship is supported by the results of our study, where PI and SS values in patients with SD are lower than those reported for normal children and remain unchanged until the end of skeletal growth.

One potential clinical implication of our findings concerns application of the PI value in planning spinal fusion in SD patients. If a lumbar fusion was planned in a mature patient with SD (for another reason than SD) the PI would not serve as a reliable guide in predicting the amount of the postoperatively desired LL. On the other hand, if correction of hyperkyphosis in a mature patient with SD was planned the decreased PI should be taken into account and a corresponding LL should be the target intraoperatively. Thus, a neutral sagittal balance could be obtained and maintained according to the formula proposed by Rose et al. [36]. The effect of corrective surgery on PI in immature individuals with SD remains unknown. Two theories seem plausible: (1) constant inhibition of increase in PI or (2) restoration of normal pelvic maturation with age related increase in PI. A limitation of our study is its retrospective design. We considered 2 groups of patients with SD, namely the skeletally immature and mature instead of 1 group followed in time from the point at which diagnosis of SD was established until skeletal maturation. However, we think that the best available scenario was used in our analysis with the 2 groups matched for SD type and sex. Another limitation of the study may be relatively small sample size of each SD group that could potentially increase LSVs in statistical analysis. In this study, PI, PT, and SS were compared between SD patients and published data for normal children, adolescents, and adults. Because of some inconsistency of data in the literature regarding the definition of TK, TLK, and LL [8, 37-41] only three pelvic parameters were compared with historically published normative data.

\section{Conclusions}

The study revealed no significant differences in the sagittal spinopelvic radiographic parameters between skeletally immature and mature subjects with SD. Pelvic incidence and SS in both groups of SD patients was significantly lower than PI and SS reported for normal children, adolescents, and adults. Thus, we suggest that pelvic incidence does not increase with skeletal maturation in patients with SD. These findings highlight the role that pelvic morphology may play as a compensatory mechanism in maintaining a neutral sagittal posture in patients with pathologic sagittal spinal alignment. Further investigations are needed to better understand the phenomenon.

\section{Conflict of interest None.}

Open Access This article is distributed under the terms of the Creative Commons Attribution License which permits any use, distribution, and reproduction in any medium, provided the original author(s) and the source are credited.

\section{References}

1. Scheuermann H (1920) Kyfosis dorsalis juvenilis. Ugeskr Laeger 82:385-393

2. Lowe TG, Line BG (2007) Evidence based medicine: analysis of Scheuermann kyphosis. Spine 32(19 Suppl):S115-S119

3. Jansen RC, van Rhijn LW, van Ooij A (2006) Predictable correction of the unfused lumbar lordosis after thoracic correction and fusion in Scheuermann kyphosis. Spine 31(11):1227-1231

4. Wood KB, Melikian R, Villamil F (2012) Adult Scheuermann kyphosis: evaluation, management, and new developments. J Am Acad Orthop Surg 20(2):113-121

5. Tardieu C, Bonneau N, Hecquet J, Boulay C, Marty C, Legaye J, Duval-Beaupère G (2013) How is sagittal balance acquired during bipedal gait acquisition? Comparison of neonatal and adult pelves in three dimensions. Evolutionary implications. J Hum Evol 65(2):209-222

6. Vrtovec T, Janssen MM, Likar B, Castelein RM, Viergever MA, Pernuš F (2012) A review of methods for evaluating the quantitative parameters of sagittal pelvic alignment. Spine $\mathrm{J}$ 12(5):433-446

7. Duval-Beaupère G, Schmidt C, Cosson P (1992) A Barycentremetric study of the sagittal shape of spine and pelvis: the conditions required for an economic standing position. Ann Biomed Eng 20(4):451-462

8. Legaye J, Duval-Beaupère G, Hecquet J, Marty C (1998) Pelvic incidence: a fundamental pelvic parameter for three-dimensional regulation of spinal sagittal curves. Eur Spine J 7(2):99-103

9. Marty C, Boisaubert B, Descamps H, Montigny JP, Hecquet J, Legaye J, Duval-Beaupère G (2002) The sagittal anatomy of the sacrum among young adults, infants, and spondylolisthesis patients. Eur Spine J 11:119-125

10. Mangione P, Gomez D, Senegas J (1997) Study of the course of the incidence angle during growth. Eur Spine J 6(3):163-167

11. Hanson DS, Bridwell KH, Rhee JM, Lenke LG (2002) Correlation of pelvic incidence with low- and high-grade isthmic spondylolisthesis. Spine 27(18):2026-2029

12. Mac-Thiong JM, Labelle H, Charlebois M, Huot MP, de Guise JA (2003) Sagittal plane analysis of the spine and pelvis in adolescent idiopathic scoliosis according to the coronal curve type. Spine 28(13):1404-1409

13. Rajnics P, Templier A, Skalli W, Lavaste F, Illés T (2002) The association of sagittal spinal and pelvic parameters in asymptomatic persons and patients with isthmic spondylolisthesis. J Spinal Disord Tech 15(1):24-30

14. Labelle H, Roussouly P, Berthonnaud E, Dimnet J, O'Brien M (2005) The importance of spino-pelvic balance in L5-s1 
developmental spondylolisthesis: a review of pertinent radiologic measurements. Spine 30(6 Suppl):S27-S34

15. Barrey C, Jund J, Perrin G, Roussouly P (2007) Spinopelvic alignment of patients with degenerative spondylolisthesis. Neurosurgery 61(5):981-986 discussion 986

16. Lim JK, Kim SM (2013) Difference of Sagittal Spinopelvic Alignments between Degenerative Spondylolisthesis and Isthmic Spondylolisthesis. J Korean Neurosurg Soc 53(2):96-101

17. Li W, Sun Z, Guo Z, Qi Q, Kim SD, Zeng Y, Sun C, Chen Z (2013) Analysis of spinopelvic sagittal alignment in patients with thoracic and thoracolumbar angular kyphosis. Spine 38(13):E813-E818

18. Jiang L, Qiu Y, Xu L, Liu Z, Wang Z, Sha S, Zhu Z (2014) Sagittal spinopelvic alignment in adolescents associated with Scheuermann's kyphosis: a comparison with normal population. Eur Spine J. doi:10.1007/s00586-014-3266-2

19. Tyrakowski M, Mardjetko S, Siemionow K (2014) radiographic spinopelvic parameters in skeletally mature patients with Scheuermann's disease. Spine 39(18):E1080-E1085

20. Sorensen K (1964) Scheuermann's juvenile kyphosis: clinical appearances, radiography, aetiology, and prognosis. Munksgaard, Copenhagen

21. Bradford DS (1977) Juvenile kyphosis. Clin Orthop Relat Res 128:45-55

22. Vedantam R, Lenke LG, Bridwell KH, Linville DL, Blanke K (2000) The effect of variation in arm position on sagittal spinal alignment. Spine 25(17):2204-2209

23. Risser JC (1958) The iliac apophysis. An invaluable sign in the management of scoliosis. Clin Orthop Relat Res 11:111-119

24. Mac-Thiong JM, Labelle H, Roussouly P (2011) Pediatric sagittal alignment. Eur Spine J 20(Suppl 5):586-590

25. Vialle R, Levassor N, Rillardon L, Templier A, Skalli W, Guigui $P$ (2005) Radiographic analysis of the sagittal alignment and balance of the spine in asymptomatic subjects. J Bone Joint Surg Am 87(2):260-267

26. Shrout PE, Fleiss JL (1979) Intraclass correlations: uses in assessing rater reliability. Psychol Bull 86:420-428

27. Keszei AP, Novak M, Streiner DL (2010) Introduction to health measurement scales. J Psychosom Res 68(4):319-323

28. Zou GY (2012) Sample size formulas for estimating intraclass correlation coefficients with precision and assurance. Stat Med 31(29):3972-3981

29. Streiner DL, Norman GR (2008) Health measurement scales a practical guide to their development and use, vol 4. Oxford University Press, Oxford
30. Wang WW, Xia CW, Zhu F, Zhu ZZ, Wang B, Wang SF, Yeung BH, Lee SK, Cheng JC, Qiu Y (2009) Correlation of Risser sign, radiographs of hand and wrist with the histological grade of iliac crest apophysis in girls with adolescent idiopathic scoliosis. Spine 34(17):1849-1854

31. Noordeen MH, Haddad FS, Edgar MA, Pringle J (1999) Spinal growth and a histologic evaluation of the Risser grade in idiopathic scoliosis. Spine 24:535-538

32. Wang S, Qiu Y, Ma Z, Xia C, Zhu F, Zhu Z (2007) Histologic, Risser sign, and digital skeletal age evaluation for residual spine growth potential in Chinese female idiopathic scoliosis. Spine 32:1648-1654

33. Lafage V, Schwab F, Patel A, Hawkinson N, Farcy JP (2009) Pelvic tilt and truncal inclination: two key radiographic parameters in the setting of adults with spinal deformity. Spine 34(17):E599-E606

34. Le Huec JC, Aunoble S, Philippe L, Nicolas P (2011) Pelvic parameters: origin and significance. Eur Spine J 20(Suppl 5):564-571

35. Vaz G, Roussouly P, Berthonnaud E, Dimnet J (2002) Sagittal morphology and equilibrium of pelvis and spine. Eur Spine $\mathrm{J}$ $1(11): 80-87$

36. Rose PS, Bridwell KH, Lenke LG, Cronen GA, Mulconrey DS, Buchowski JM, Kim YJ (2009) Role of pelvic incidence, thoracic kyphosis, and patient factors on sagittal plane correction following pedicle subtraction osteotomy. Spine 34(8):785-791

37. Bernhardt M, Bridwell KH (1989) Segmental analysis of the sagittal plane alignment of the normal thoracic and lumbar spines and thoracolumbar junction. Spine 14:717-721

38. Boseker EH, Moe JH, Winter RB, Koop SE (2000) Determination of "normal" thoracic kyphosis: a roentgenographic study of 121 " "normal" children. J Pediatr Orthop 20:796-798

39. Cil A, Yazici M, Uzumcugil A, Kandemir U, Alanay A, Alanay Y, Acaroglu RE, Surat A (2005) The evolution of sagittal segmental alignment of the spine during childhood. Spine 30:93-100

40. Barrey C, Jund J, Noseda O, Roussouly P (2007) Sagittal balance of the pelvis-spine complex and lumbar degenerative diseases. A comparative study about 85 cases. Eur Spine J 16(9):1459-1467

41. Boulay C, Tardieu C, Hecquet J, Benaim C, Mouilleseaux B, Marty C, Prat-Pradal D, Legaye J, Duval-Beaupère G, Pélissier J (2007) Sagittal alignment of spine and pelvis regulated by pelvic incidence: standard values and prediction of lordosis. Eur Spine J 15(4):415-422 